submitted version

\title{
Scales for co-compact embeddings of virtually free groups
}

\author{
Udo Baumgartner \\ School of Mathematical and Physical Sciences, The University of Newcastle, University Drive, Building V, \\ Callaghan, NSW 2308, Australia \\ e-mail: Udo.Baumgartner@newcastle.edu. au
}

February 8, 2020

\begin{abstract}
Let $\Gamma$ be a group which is virtually free of rank at least 2 and let $\mathcal{F}_{t d}(\Gamma)$ be the family of totally disconnected, locally compact groups containing $\Gamma$ as a co-compact lattice. We prove that the values of the scale function with respect to groups in $\mathcal{F}_{t d}(\Gamma)$ evaluated on the subset $\Gamma$ have only finitely many prime divisors. This can be thought of as a uniform property of the family $\mathcal{F}_{t d}(\Gamma)$.
\end{abstract}

Key words uniform lattice, virtually free group, totally disconnected group, scale function

Mathematics Subject Classification (2000): 22D05, 57M07, 20E08

\section{Introduction}

Let $\Gamma$ be a finitely generated group and let $S$ be a finite set of generators for $\Gamma$. The group $\Gamma$ embeds as a cocompact, discrete subgroup in the automorphism group of its Cayley graph with respect to $S$, which is a totally disconnected, locally compact group.

This note begins the examination of common features of the class $\mathcal{F}(\Gamma)$ of all embeddings of a fixed finitely generated group $\Gamma$ as a discrete, cocompact subgroup in some locally compact group. By the previous paragraph, the automorphism group of any Cayley graph of $\Gamma$ belongs to $\mathcal{F}(\Gamma)$. In this article we treat the case where $\Gamma$ is virtually free of finite rank at least 2. Most of our results will be about the subclass $\mathcal{F}_{t d}(\Gamma)$ of $\mathcal{F}(\Gamma)$ consisting of cocompact embeddings of $\Gamma$ into totally disconnected, locally compact groups; see however Corollary 3.

This program was suggested (in a less general form) by George Willis in [Wil04, penultimate topic in Section 6]. One of the common features of the class $\mathcal{F}_{t d}(\Gamma)$ suggested there for examination is the set of values of the scale function, whose definition we recall below, with respect to members of $\mathcal{F}_{t d}(\Gamma)$ evaluated on $\Gamma$.

Furman undertook a related project for lattices in semisimple (connected real) Lie groups in [Fur01]. He proposed to classify all second countable, locally compact groups, which admit a lattice embedding (not necessarily cocompact) of a given lattice $\Gamma$ in a semisimple connected, real 
Lie group ([Fur01, p. 31]) and solved this problem if $\Gamma$ is a lattice in a simple Lie group of higher rank ([Fur01], Theorem A). He was also able to classify second countable, locally compact groups which admit lattice embeddings which are cocompact, if $\Gamma$ is an irreducible lattice in either a semisimple, connected, real Lie group not locally isomorphic to $\mathrm{SL}_{2}(\mathbb{R})$ or a cocompact lattice in a group locally isomorphic to $\mathrm{SL}_{2}(\mathbb{R})$ ([Fur01], Theorem B and Theorem C).

We now return to the question, how one might characterize common features of discrete, cocompact embeddings of a given group $\Gamma$ into some totally disconnected, locally compact group. One way in which this may be done, following the suggestion by Willis mentioned above, is by restricting the values taken by the scale functions with respect to the codomain of the embeddings on the image of $\Gamma$.

For a totally disconnected, locally compact group $G$, the value of the scale function $s_{G}$ at an automorphism, $\alpha$, of $G$ measures minimal distortion of compact, open subgroups of $G$ under $\alpha$. The scale function is defined on the set of automorphisms of $G$ by the formula

$$
s_{G}(\alpha):=\min \{|\alpha(V): \alpha(V) \cap V|: V \text { a compact, open subgroup of } G\} .
$$

Note that the minimum above is attained, because it is formed for a set of positive integers. A compact, open subgroup $O$ of $G$ is tidy for $\boldsymbol{\alpha}$ if this minimum is attained at $O$. The scale of a group element, $x$, of $G$ is defined to be the value of the scale function with respect to $G$ at conjugation by $x$.

The collection of tidy subgroups for an automorphism and the scale function are invariants, which have been used to answer various questions on totally disconnected, locally compact groups; see [Wil04] for a survey.

The scale of an automorphism of a locally compact, totally disconnected group is an analogue for the set of eigenvalues of a linear transformation. That there should be any uniform bound on the primes dividing values of the scale with respect to the elements of $\mathcal{F}_{t d}(\Gamma)$ is not clear, even though a single element of $\mathcal{F}_{t d}(\Gamma)$ contributes only a finite number of prime factors by Theorem 3.4 in [Wil01b].

The author was surprised to discover, that even a slightly stronger result can be proved quite easily in the case when $\Gamma$ is virtually a free group of rank at least 2 , by putting together work by Lee Mosher, Michah Sageev, and Kevin Whyte and Alexander Lubotzky; see Corollary 11.

It can be shown, that virtually abelian groups embed cocompactly only in totally disconnected, locally compact groups whose scale function is identically 1 ; hence a result analogous to Corollary 11 does hold for virtually infinite cyclic groups also. As a further remark, by the results of Furman mentioned above, groups $\Gamma$ to which one of his Theorems A, B, or C applies, also embed cocompactly only in a totally disconnected, locally compact group $G$ whose scale function is identically 1 , provided the group $G$ is second countable.

The method of proof used in this note suggests, that replacing the use of Theorem 1 by an appeal to the main result of [Ahl02] will prove a generalization of Corollary 11 to groups $\Gamma$, which are quasi-isometric to a product of finitely many trees once a replacement for Lemma 9 can be found, to bound the quotient of an action of a group $\Gamma$ on a product of trees in terms of properties of $\Gamma$.

Note that the class of groups which are quasi-isometric to products of finitely many trees not only contains products of virtually free groups but also the finitely presented simple groups constructed by Burger and Mozes; see [BM97]. 


\section{Conventions and outline of the paper}

In what follows, $\Gamma$ will denote a group which is virtually free of finite rank at least 2 , while $F$ will denote a free group of finite rank at least 2. We will first treat the case of free groups to obtain Theorem 10, which gives more precise information in this special case. Then we will deduce the announced result for virtually free groups, Corollary 11, as a corollary.

To ease discussion of the circle of ideas which form the topic of this paper, we adapt the following terminology. Let $\Lambda$ and $G$ be a locally compact groups. In our applications, $\Lambda$ will usually be finitely generated and discrete. An injective homomorphism $\varphi: \Lambda \rightarrow G$ such that $\varphi(\Lambda)$ is a closed, cocompact subgroup in $G$ will be called an envelope of $\Lambda$. The group $G$ will also be called an envelope of $\Lambda$ by abuse of language; any envelope of a compactly generated group is compactly generated.

In Section 3 we will use a quasi-isometric rigidity result by Lee Mosher, Michah Sageev, and Kevin Whyte to see that any envelope of $\Gamma$ acts cocompactly on a locally finite tree. From this we deduce in Section 4 that, in terms of scale values, $\Gamma$ has a larger envelope which is an automorphism group of a locally finite tree. Specializing to free groups in Section 5 and using Lubotzky's results on Schottky groups of automorphisms of trees, we bound the geometry of the underlying trees. The required results are then immediate consequences.

In this note we adhere to the following conventions: 0 is a natural number. Graphs may have loops and multiple edges, but edges will not be given an orientation, except on occasion of applications of the Bass-Serre theory of groups acting on trees. The reader may consult [Ser80], section 2.1 for a formal setup of the terminology and notation for graphs. An isometry of a tree will be called elliptic or hyperbolic according to whether it admits a fixed point (which may be a geometric edge) or not. Conjugation by a group element $g$ is understood to be the map $x \mapsto g x g^{-1}$. The relations $\subset, \triangleleft$ etc. always imply strict inclusion. Any automorphism of a topological group will be assumed to be a homeomorphism.

\section{Envelopes of virtually free groups of finite rank act on bushy trees}

Any envelope $G$ of a finitely generated group $\Lambda$ quasi-acts on each of the Cayley graphs of $\Lambda$. If $\Gamma$ is virtually free of finite rank at least 2, more can be said, thanks to the following theorem by Lee Mosher, Michah Sageev, and Kevin Whyte. The theorem shows, that any envelope $G$ of $\Gamma$ is a compact extension of a cocompact subgroup of the automorphism group of a locally finite bushy tree $T$ (a tree $T$ is bushy if each point of $T$ is a uniformly bounded distance from a vertex having at least 3 unbounded complementary components). This will enable us to reduce to the case where the envelope $G$ is the group Aut $(T)$, for such a tree $T$ in the next section.

Theorem 1 ([MSW03, Theorem 9]). Let $G$ be a locally compact topological group which contains a cocompact lattice which is virtually free of finite rank at least 2 . Then there exists a cocompact action of $G$ on a bushy tree $T$ of bounded valence, inducing a continuous, closed homomorphism $\tau: G \rightarrow \operatorname{Aut}(T)$ with compact kernel and cocompact image.

Remark 2.

1. The statement of Theorem 9 on page 125 in [MSW03] uses the word 'proper' in place of 'closed'. The proof of Theorem 9 on page 161 in [MSW03] makes it clear, that the intended meaning of proper in this context is closed. 
2. In Theorem 1 we can assume that the action of $G$ on $T$ via $\tau$ is minimal, replacing $T$ with the minimal $G$-invariant subtree if necessary. Indeed, the $G$-action on the tree constructed in the course of the proof of Theorem 1 in [MSW03] is already minimal. As we are only interested in groups containing lattices of positive rank, such a minimal tree will have no vertex of degree 1 .

3. By Theorem 16 in [Möl], totally disconnected envelopes of a group which is virtually free of finite rank can be characterized as those groups whose rough Cayley graphs are quasi-isometric to a tree. That tree is bushy if and only if the rank of free subgroups of the cocompact discrete subgroup are at least 2 .

Corollary 3. Let $G$ be an envelope of a virtually free group of finite rank at least 2 . Then the connected component of the identity of $G$ is compact.

Proof. By Theorem 1, $G$ has a continuous homomorphism $\tau$ into the automorphism group of a locally finite tree $T$ with compact kernel. Since Aut $(T)$ is totally disconnected, the connected component $G_{0}$ of the identity in $G$ is contained in the kernel of $\tau$. We conclude that $G_{0}$ is relatively compact, being contained in $\operatorname{ker}(\tau)$. Since $G_{0}$ is closed as well, we conclude that $G_{0}$ is compact as claimed.

\section{Scale function on envelopes which are automorphism groups of bushy trees}

The next three results will show, that values of the scale function on totally disconnected envelopes of the free group $F$ can be bounded in terms of values of the scale function on envelopes of $F$ which are automorphism groups of some locally finite, bushy tree $T$.

First, we show that whenever $G$ is an envelope of $F$, then the codomain of the map $\tau$, introduced in Theorem 1, is also an envelope of $F$.

Proposition 4. Let $\varphi: F \rightarrow G$ be an envelope of a free group $F$ of finite rank at least 2 . Let $\tau$ be the homomorphism from $G$ into the automorphism group of the tree $T$ provided by Theorem 1 and let $\theta: F \rightarrow$ Aut $(T)$ be the composite of $\varphi$ and $\tau$. Then $\theta$ is injective and $\theta(F)$ is a cocompact lattice in Aut $(T)$. Hence Aut $(T)$ is an envelope of $F$.

Proof. The group $\theta(F)$ is a discrete subgroup of Aut $(T)$, because the kernel of $\tau$ is compact. The kernel of $\theta$ is a compact subgroup of the discrete group $F$, hence is finite. Since we are assuming that $F$ is a free group, it is torsion free and we conclude that the kernel of $\theta$ is trivial and $\theta$ is injective.

Since $F$ is cocompact in $G$ and $\tau(G)$ is cocompact in Aut $(T)$ we conclude that $\theta(F)$ is cocompact in $\operatorname{Aut}(T)$. We have verified all parts of our claim.

The next lemma shows that the scale of an element does not change if we apply a homomorphism, which is a perfect map (which, for a homomorphism of groups, means a continuous, open, surjective homomorphism with compact kernel).

Lemma 5. Let $\pi: G \rightarrow \widehat{G}$ be a continuous, open, surjective homomorphism with compact kernel between totally disconnected, locally compact groups. Let $\alpha$ be an automorphism of $G$ preserving $\operatorname{ker}(\pi)$ and $\widehat{O}$ a subgroup of $\widehat{G}$ tidy for the automorphism $\widehat{\alpha}$ induced by $\alpha$ on $\widehat{G}$. Then the group $\pi^{-1}(\widehat{O})$ is tidy for $\alpha$ and $s_{G}(\alpha)=s_{\widehat{G}}(\widehat{\alpha})$. 
Proof. Put $O:=\pi^{-1}(\widehat{O})$. Since $\pi$ is continuous and the kernel of $\pi$ is compact, the group $O$ is compact and open. The conclusion follows from the definition of the scale of $\alpha$ as a minimum, the equation

$$
|\alpha(O): \alpha(O) \cap O|=|\widehat{\alpha}(\widehat{O}): \widehat{\alpha}(\widehat{O}) \cap \widehat{O}|=s_{\widehat{G}}(\widehat{\alpha})
$$

and Proposition 4.7 in [Wil01a].

The larger envelope of $F$ obtained from Proposition 4 does not have smaller scale values, as shown by the next result.

Corollary 6. Let $G$ be a totally disconnected, locally compact group, $T$ a locally finite, bushy tree and $\tau: G \rightarrow \operatorname{Aut}(T)$ a continuous, closed homomorphism with compact kernel. Then

$$
s_{G}(x) \leq s_{\text {Aut }(T)}(\tau(x)) \quad \text { for all } x \in G .
$$

Proof. Let $x$ be an element of $G$. Put $\widehat{G}:=\tau(G)$, let $\pi: G \rightarrow \widehat{G}$ be the map induced by $\tau$ and let $\alpha$ be conjugation by $x$. Then $\pi$ and $\alpha$ satisfy the conditions on the maps with the same names in Lemma 5 and $\widehat{\alpha}$ is conjugation by $\pi(x)$. We conclude that $s_{G}(x)=s_{\widehat{G}}(\pi(x))$.

Furthermore, renaming $\alpha$ as conjugation by $\tau(x)$ and applying Proposition 4.3 in [Wil01a] with $H:=\widehat{G}$, we deduce that $s_{\widehat{G}}(\pi(x)) \leq s_{\text {Aut }(T)}(\tau(x))$.

Combining these two relations we obtain $s_{G}(x) \leq s_{\text {Aut }(T)}(\tau(x))$. Since the choice of $x$ was arbitrary, the claim follows.

The scale function of a closed subgroup, $G$, of the automorphism group of a locally finite tree, $T$, can be determined geometrically, as seen in the next result, Lemma 7 .

The geometric description of the value of the scale function with respect to $G$ at a hyperbolic isometry, $h$ say, in $G$ given in Lemma 7 uses ramification indices of a subtree $T_{G, \epsilon}$ of $T$, which depends on the group $G$ and the attracting end, $\epsilon$, of $h$. The tree $T_{G, \epsilon}$ is defined as follows: Given an end $\epsilon$ of $T$ the tree $T_{G, \epsilon}$ is the union of the axes of all hyperbolic isometries in $G_{\epsilon}$.

Lemma 7 ([BW06, Lemmas 26 and 31]). Let $G$ be a closed subgroup of the automorphism group of a locally finite tree $T$.

1. Let $g$ be an elliptic isometry of $T$ in $G$. Then $g$ is topologically periodic and $s_{G}(g)=1=s_{G}\left(g^{-1}\right)$.

2. Let $h$ be a hyperbolic isometry of $T$ in $G$ with attracting end $\epsilon$, of translation length $n$ say. Let $q_{1}+1, \ldots, q_{n}+1$ be the ramification indices of $n$ consecutive vertices on the axis of $h$ with respect to the tree $T_{G, \epsilon}$. Then $s_{G}(h)=\prod_{i=1}^{n} q_{i}$.

The geometric description of the scale function given by Lemma 7 will be useful in the next section.

\section{Upper bounds for scales on envelopes of Schottky lattices}

The isomorphic image $\theta(F)$ of $F$ obtained in Proposition 4, is a finitely generated, torsion free, discrete subgroup of Aut $(T)$. A finitely generated, torsion free, discrete subgroup of the automorphism group of a tree will be called a Schottky subgroup of Aut $(T)$. This terminology was coined by Alexander Lubotzky in section 1 of the paper [Lub91], where he describes the structure of such groups.

Let $\Gamma$ be a Schottky subgroup of Aut $(T)$. Then $\Gamma$ acts freely on $T$ and hence is a free group; it has finite rank, because it is finitely generated by assumption. Bass-Serre theory ([Ser80], chapter I 
or [Bas93]) provides us with a nice basis of $\Gamma$, via its identification with the fundamental group of the trivial graph of groups on the quotient graph $X:=\Gamma \backslash T$.

Any basis of a Schottky subgroup, $\Gamma$, of Aut $(T)$ that is obtained from Bass-Serre theory in the manner explained below will be called a Schottky basis in what follows. We recall now how these bases of $\Gamma$ are obtained, following the proof of Proposition 1.7 in [Lub91]. This construction of Schottky bases for the group $\Gamma$ will involve several choices, only some of which will be of interest for us later; see Lemma 8.

Choose an orientation of the edges of $X$. Any choice of a maximal subtree, $Y$ say, in $X:=\Gamma \backslash T$ defines the set of edges $X \backslash Y$, belonging to $X$ but not to $Y$, which freely generate the fundamental group of the graph $X$. The set of edges $X \backslash Y$ corresponds to some set of isometries of the universal covering tree $T$ of $X$. This correspondence is defined in two stages.

In the first stage defining this correspondence, choose connected subgraphs $Y_{T} \subseteq X_{T}$ in $T$ such that the canonical projection, $p: T \rightarrow X$, is bijective on the set of vertices of $Y_{T}$ and the set of edges of $X_{T}$. Make this choice in such a way that the origins of all the edges of $X_{T} \backslash Y_{T}$ belong to $X_{T}$. Such a pair $\left(Y_{T}, X_{T}\right)$ is called a 'lifting' or 'opening' of $X$ in the literature. For later use, denote the inverse image of a vertex, $v$, in $Y$ under $p$ that is contained in $Y_{T}$ by $\widetilde{v}$ and the inverse image of an edge, $e$, in $X$ under $p$ that is contained in $X_{T}$ by $\widetilde{e}$. Also, for an edge, $e$, denote by $o(e)$ and $t(e)$ the origin and terminal vertices of $e$. For the second stage defining the set of isometries corresponding to $X \backslash Y$ choose, for each edge $e$ in $X \backslash Y$, an element, $\gamma_{e}$, of $\Gamma$ such that, $t(\widetilde{e})=\gamma_{e} \cdot \widetilde{t(e)}$.

This construction is illustrated in a simple example below, where $\Gamma$ is the free group on 3 generators. To the left, we see some possible quotient graph $\Gamma \backslash T$. The choice of a maximal subtree, $Y$, in $\Gamma \backslash T$ is indicated by labeling the edges outside $Y$ by the symbols $e_{1}, e_{2}$ and $e_{3}$. To the right, we see how the graph $X_{T}$ of an opening of the graph $\Gamma \backslash T$ determined by the choice of orientation and maximal subtree looks like. Only the orientation of the edges $e_{1}, e_{2}$ and $e_{3}$ matters, and is indicated by arrows in both pictures. The vertices of the graph $X_{T}$, that do not belong to the graph $Y_{T}$, are indicated by hollow circles. All other vertices in both pictures are indicated by filled circles.
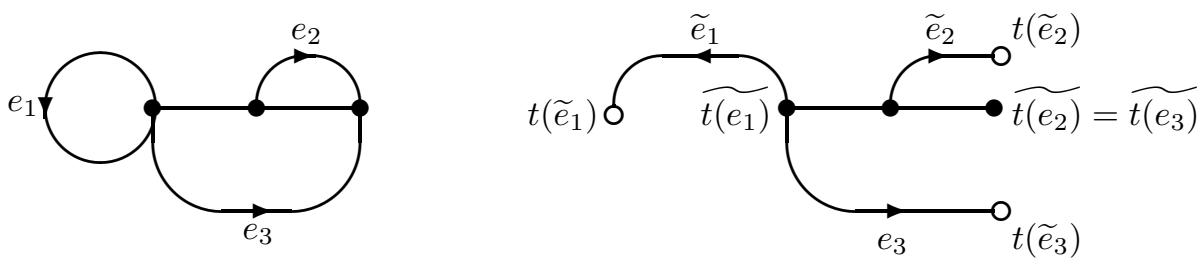

The set $\left\{\gamma_{e}: e \in X \backslash Y\right\}$, which we obtain from all the choices we made, is a free set of generators of $\Gamma$ by [Ser80, §5], which we call the Schottky basis of $\Gamma$ determined by these choices.

The Schottky basis $\left\{\gamma_{e}: e \in X \backslash Y\right\}$ consists of hyperbolic isometries of $T$. This is true because the set of edges of the graph $X_{T}$ is a fundamental domain for the $\Gamma$-action on the edges. The latter argument works for any set $\left\{\gamma_{e}: e \in X \backslash Y\right\}$ of elements of a group $\Gamma$ constructed in the way described above, as long as $\Gamma$ acts without inversion of edges. In the case at hand, where $\Gamma$ is a free group, it may alternatively be seen, by using that $\Gamma$ must act freely on $X$.

Below, we will need the following information on the axes (and translation lengths) of elements in the Schottky basis $\left\{\gamma_{e}: e \in X \backslash Y\right\}$. The axis of the element $\gamma_{e}$ passes through the vertices $\widetilde{t(e)}$ and $t(\widetilde{e})=\gamma_{e} . \widetilde{t(e)}$, whose distance is therefore the translation length of $\gamma_{e}$. This may be seen by applying Lemma 1.2 in [Lub91] with $\gamma$ equal to $\gamma_{e}, x$ equal to $\widetilde{t(e)}$ and $y$ equal to the first vertex 
on the shortest path joining $\widetilde{t(e)}$ to $t(\widetilde{e})$, (which, by the way, is contained in $X_{T}$ ). The axis of $\gamma_{e}$ also passes through $o(\widetilde{e})$, because by our choice of a lift for $e$, the vertex $t(\widetilde{e})$ is incident to only one edge of $X_{T}$.

This finishes the description of the construction of the set of Schottky bases for $\Gamma$, save for two final remark for readers familiar with [Lub91]. First, in [Lub91], a Schottky basis is supposed to satisfy an apparently stronger condition than the one we have verified here, see Definition 1.4 in [Lub91]. But, as stated in the proof of Propostion 1.7 in [Lub91], the required labeling of the axes of the elements in the basis $\left\{\gamma_{e}: e \in X \backslash Y\right\}$ is obtained if we choose, for every $e \in X \backslash Y$, the labeling of the axis of $\gamma_{e}$ determined by labeling $\widetilde{t(e)}$ with the symbol $x_{1}$. Second, the construction above gives all Schottky bases for $\Gamma$ in the sense defined in Definition 1.4 in [Lub91]. To see this, note that the fundamental domain $F$ from Proposition 1.6 in [Lub91] defines an opening for $\Gamma \backslash T$, such that the given Schottky basis $\left\{\gamma_{1}, \ldots, \gamma_{l}\right\}$ is one of the possible Schottky bases obtainable from that opening in the sense and the way described above.

The next result is obvious from the discussion of the construction of a Schottky basis above.

Lemma 8. Let $T$ be a tree. Suppose that $\left\{\gamma_{1}, \ldots, \gamma_{l}\right\}$ and $\left\{\gamma_{1}^{\prime}, \ldots, \gamma_{l}^{\prime}\right\}$ are two Schottky bases of the same Schottky subgroup, $\Gamma$, in Aut $(T)$, obtainable from the same choice of maximal subtree, $Y$ say, in $\Gamma \backslash T$. Let $\left\{e_{1}, \ldots, e_{l}\right\}$ be the set of edges of $\Gamma \backslash T$ outside $Y$. Then there are permutations, $\pi$ and $\pi^{\prime}$, of the set $\{1, \ldots, l\}$ such that for each integer $i$ with $1 \leq i \leq l$ the restriction of the canonical projection $p: T \rightarrow \Gamma \backslash T$ induces bijections from (1) onto (0) and from (1') onto (0) where:

(0) is the set of vertices on the shortest path in $Y$ connecting the two vertices of the edge $e_{i}$;

(1) is the set of vertices of a fundamental domain for the vertices on the axis of $\gamma_{\pi(i)}$ modulo $\left\langle\gamma_{\pi(i)}\right\rangle$;

$\left(1^{\prime}\right)$ is the set of vertices of a fundamental domain for the vertices on the axis of $\gamma_{\pi^{\prime}(i)}^{\prime}$ modulo $\left\langle\gamma_{\pi^{\prime}(i)}^{\prime}\right\rangle$.

Let $T$ be a tree and $\Gamma$ be a Schottky subgroup of Aut $(T)$. In what follows, we will only be interested in those properties of a Schottky basis, that are already determined by the quotient graph $\Gamma \backslash T$ and the set of collections of vertices described in (0), indexed by the set $\left\{e_{1}, \ldots, e_{l}\right\}$ of edges outside a maximal subtree, $Y$, of $\Gamma \backslash T$. Therefore, we will allow ourselves to speak, on occasion, somewhat inaccurately, of 'the Schottky basis determined by the maximal subtree $Y$ '.

We now return to the Schottky subgroup $\theta(F)$ of Aut $(T)$ obtained in Proposition 4 . The group $\theta(F)$ is also a (cocompact) lattice in Aut $(T)$. It follows that the quotient graph $X:=\theta(F) \backslash T$ is finite. Using part 2 of Remark 2, we may assume that $T$ has no vertex of degree 1 . This implies that $X$ has no vertex of degree 1 either.

We will call a Schottky subgroup of Aut $(T)$ that is also a lattice a Schottky lattice. We now derive our main lemma on Schottky lattices.

Lemma 9. Any tree $T$ with all vertices having degree at least 3, which admits a Schottky lattice on $n$ generators, belongs to a finite list of universal covering trees of finite graphs. In particular there are integers $K_{n}$ and $l_{n}$, such that

1. the ramification indices of $T$ are at most $K_{n}$;

2. every member of a Schottky basis of a Schottky lattice on $T$ has translation length at most $l_{n}$.

Proof. It suffices to show, that there are only finitely many graphs with fundamental group free of rank $n$ with all vertices of degree at least 3 . We will show this, by deriving upper bounds for the number of vertices and edges of such a graph. 
Let $X$ be such a graph. It has $n$ edges outside a maximal subtree. Denote by $e$ the total number of edges and by $v$ the number of vertices of $X$. Since the number of vertices of a tree exceeds the number of its edges by one, we have $n=e-v+1$.

The sum of the degrees of vertices in $X$ is at most $2 e$, because at most 2 different vertices belong to any edge. On the other hand that sum is at least $3 v$ by our assumption on the vertex degrees. It follows that $e \geq 3 / 2 v$. Substituting this inequality into the equation stated in the last paragraph we obtain the upper bound $v \leq 2(n-1)$ for $v$ in terms of $n$.

The equation $n=e-v+1$ implies that $e=n+v-1$, which is seen to be bounded above by $3(n-1)$ using the upper bound $v \leq 2(n-1)$, derived above. This completes the proof.

Finally, we derive our main result.

Theorem 10. Let $F$ be a free group of finite rank at least 2. There is a constant $V(F)$ such that for all envelopes $\varphi: F \rightarrow G$ of $F$ into a totally disconnected group, there is a free generating set $S_{\varphi}$ in $F$ such that

$$
\prod_{t \in S_{\varphi}} s_{G}(\varphi(t)) \leq V(F) .
$$

Furthermore, the set of primes dividing one of the numbers

$$
s_{G}(x) ; \quad G \text { a F-envelope, } x \in G
$$

is finite.

Proof. Proposition 4 and Corollary 6 show that, for both claims, we may assume that $G$ runs through automorphism groups of bounded valence, bushy trees, which are envelopes of $F$. As pointed out in Remark 2, we may assume that the corresponding tree actions are minimal, hence that none of these tree has a vertex of degree 1 . Since $F$ is torsion free, it acts on the trees in an orientation preserving way. Hence, we may also assume that none of the trees has vertices of degree 2 .

As explained at the beginning of this section, this implies that the image of $F$ under $\varphi$ is a Schottky lattice. We choose $S_{\varphi}$ to be a Schottky basis of $\varphi(F)$. It follows then from Lemma 7 and Lemma 9 that we have $V(F) \leq\left(K_{n}-1\right)^{n l_{n}}$ and that no prime divisor of $s_{\text {Aut }(T)}(x)$ can exceed $K_{n}$. This shows our claim.

The second property in the above theorem can also be obtained for groups which are just virtually free of finite rank at least 2 .

Corollary 11. Let $\Gamma$ be virtually a free group of finite rank at least 2 . Then the set of primes dividing one of the numbers

$$
s_{G}(x) ; \quad G \text { a totally disconnected } \Gamma \text {-envelope, } x \in G
$$

is finite.

Proof. Let $F$ be a free subgroup of finite index in $\Gamma$ and let $\iota: F \hookrightarrow \Gamma$ be the inclusion of $F$ in $\Gamma$. If $\varphi: \Gamma \rightarrow G$ is a $\Gamma$-envelope, then the composite $\varphi \circ \iota: F \rightarrow G$ is an $F$-envelope. Therefore the claim follows immediately from the second statement of Theorem 10.

We now make terminology and notation available, to describe the phenomenon established for free groups of finite rank at least 2 in the first part of Theorem 10. This terminology and notation will be used in the next section. 
Definition 12. Let $\Lambda$ be a finitely generated group and let $\varphi: \Lambda \rightarrow G$ be an envelope of $\Lambda$ with totally disconnected, locally compact codomain $G$.

1. If $S$ is a finite set of generators for $\Lambda$, the number $\operatorname{vol}_{\varphi}(S):=\prod_{t \in S} s_{G}(\varphi(t))$ will be called the scale volume of $S$ with respect to $\varphi$.

2. The number $\operatorname{vol}_{\varphi}\langle\Lambda\rangle:=\min \left\{\operatorname{vol}_{\varphi}(S): S\right.$ is finite and generates $\left.\Lambda\right\}$ will be called the scale volume of $\Lambda$ with respect to $\varphi$.

3. The number $s-\operatorname{vol}(\Lambda):=\sup \left\{\operatorname{vol}_{\varphi}\langle\Lambda\rangle: \varphi\right.$ is an envelope of $\Lambda$ with totally disconnected codomain $\}$ (which may be infinite) will be called the scale volume of $\Lambda$.

Using the terminology just introduced, the first part of Theorem 10 may be restated as 'the scale volume of a free group of finite rank at least 2 is finite'.

\section{Explicit bounds on scales}

In this section we will address the problem of getting quantitative versions of Theorem 10 and Corollary 11. We start with explicit bounds on possible values of the scale function on a free group of finite rank at least 2 .

Proposition 13. Let $n$ be an integer which is at least 2 . Let $\mathcal{B}(n)$ be the class of all graphs with all vertices of degree at least 3 and fundamental group free of rank $n$. Then

1. The maximal vertex degree of graphs in $\mathcal{B}(n)$ is $2 n$. This maximal degree is achieved for the unique graph, $B_{2 n}$, in $\mathcal{B}(n)$ with 1 vertex and is not achieved for any other graph in $\mathcal{B}(n)$. The fundamental group, $F$, of the trivial graph of groups over $B_{2 n}$ admits, via its action on the universal covering tree, $T_{2 n}$, of $B_{2 n}$, which is a homogenous tree of degree $2 n$, a discrete, cocompact embedding into Aut $\left(T_{2 n}\right)$. All elements of the unique Schottky basis of $F$ obtained using all edges of $B_{2 n}$ have translation length 1 and the scale function of $\operatorname{Aut}\left(T_{2 n}\right)$ assumes the value $2 n-1$ on all of these elements.

2. For any odd integer $s$ with $3 \leq s \leq 2 n-1$, there is a graph, $B(s)$, in $\mathcal{B}(n)$ such that the scale function on the automorphism group of the covering tree, $T(B(s))$, of $B(s)$ assumes the value $s$ on some hyperbolic isometry of translation length 1 which belongs to every Schottky basis of the fundamental group, $F$, of the trivial graph of groups over $B(s)$. The group $F$ admits, via its action on $T(B(s))$, a discrete, cocompact embedding into Aut $(T(B(s)))$.

3. Let $n$ be an integer which is at least 2. The maximal translation length of a member of a Schottky basis for the image of the free group on $n$ generators in a discrete, cocompact embedding in the automorphism group of a locally finite tree all of whose vertices have degree at least 3 is $2(n-1)$. More precisely, there is a graph, $B^{2(n-1)}$, in $\mathcal{B}(n)$ such that every Schottky basis of the fundamental group, $F$, of the trivial graph of groups over $B^{2(n-1)}$ has an element whose translation length is $2(n-1)$ and whose scale with respect to the automorphism group of the covering tree, $T\left(B^{2(n-1)}\right)$, of $B^{2(n-1)}$ is $2^{2(n-1)}$. The group $F$ admits, via its action on $T\left(B^{2(n-1)}\right)$, a discrete, cocompact embedding into $\operatorname{Aut}\left(T\left(B^{2(n-1)}\right)\right)$.

Proof. Proof of 1: In proving the first claim, we start by confirming the statements made in the first two sentences thereof. Thanks to the lower bound on vertex degrees for graphs is $\mathcal{B}(n)$, the following holds for any graph, $B$ say, in $\mathcal{B}(n)$. Contracting an edge of a maximal subtree in $B$ to its initial vertex, $o$ say, creates another graph in $\mathcal{B}(n)$, whose vertex degree at $o$ is strictly larger than the vertex degree of $o$ in $B$. Therefore, the maximal vertex degree of a graph in $\mathcal{B}(n)$ is achieved 
for a graph with just one vertex. Since there is a unique graph, $B_{2 n}$, in $\mathcal{B}(n)$ with one vertex, we have seen that the first two sentences are true.

A moment's thought shows, that all the remaining statements made in the first claim save the value taken by the scale function of Aut $\left(T_{2 n}\right)$ on the elements of the Schottky basis of $F$ follow immediately from the statements just proved.

To see that this remaining statement is true also, it suffices by Lemma 7 to show that for any end $\epsilon$ of $T_{2 n}$ the tree $\left(T_{2 n}\right)_{\text {Aut }\left(T_{2 n}\right), \epsilon}$ (introduced in the paragraph preceding Lemma 7, page 5 ) is the whole tree $T_{2 n}$. Since for any two distinct ends, $\epsilon$ and $\epsilon^{\prime}$ say, in a homogenous tree such as $T_{2 n}$ there is a hyperbolic isometry (of translation length 1) whose axis is the line joining $\epsilon$ and $\epsilon^{\prime}$, we clearly have $\left(T_{2 n}\right)_{\text {Aut }\left(T_{2 n}\right), \epsilon}=T_{2 n}$, for any end $\epsilon$, and we finished the proof of our first claim.

Proof of 2: To obtain the statements of the second claim, we first introduce the graph $B(s)$ for a given odd integer $s$ with $3 \leq s \leq 2 n-1$. If $s$ is equal to $2 n-1$, then, by the first claim, which we already proved, the graph $B_{2 n}$ has all the properties the graph $B(2 n-1)$ is required to satisfy. Therefore, we may assume in what follows, that $s$ is at most $2 n-3$.

If $s \leq 2 n-3$, the graph $B(s)$ has 2 vertices, $v_{0}$ and $v_{1}$. The vertices $v_{0}$ and $v_{1}$ are joined by one, respectively two edges depending on whether $s$ is different from or equal to $n-1$. No matter what the value of $s$ is, attach $(s+1) / 2 \geq 2$ loops to the vertex $v_{0}$. If $s \neq n-1$, attach $n-(s+1) / 2 \geq n-(2 n-2) / 2=1$ loops to the vertex $v_{1}$. If $s=n-1$, attach $n-n / 2-1=n / 2-1 \geq 1$ loops to the vertex $v_{1}$.

For each odd integer $s$ with $3 \leq s \leq 2 n-3$ the graph $B(s)$ belongs to $\mathcal{B}(n)$ by construction. The degrees $d_{0}$ and $d_{1}$ of the vertices $v_{0}$ and $v_{1}$ of $B(s)$ are different.

We claim that the scale function with respect to Aut $(T(B(s)))$ assumes the value $s$ on each of the elements of $F$ obtained from the $(s+1) / 2$ loops based at $v_{0}$. To see this, let $h$ be one of the elements of $F$ obtained from loops at $v_{0}$ and let $\epsilon$ be the attracting end of $h$. We will determine the tree $T(B(s))_{\operatorname{Aut}(T(B(s))), \epsilon}$.

Note that all vertices on the axis of $h$ are translates of a lift of $v_{0}$ and hence have degree $d_{0}$. The vertices on the axis of every hyperbolic isometry of $T(B(s))$ that has $\epsilon$ as attracting end must have degree $d_{0}$ also. Let $k$ be such a hyperbolic isometry. By the above observation on the degrees of vertices on the axis of $k$, this axis maps to a closed edge path at $v_{0}$ in $B(s)$ all of whose edges belong to the subgraph, $B\left(v_{0}\right)$, of $B(s)$ consisting of the vertex $v_{0}$ and all the loops based at $v_{0}$. Conversely, every closed edge path at $v_{0}$ can be obtained as an image of a hyperbolic isometry of the tree $T(B(s))$ fixing the end $\epsilon$. We conclude that the tree $T(B(s))_{\text {Aut }(T(B(s))), \epsilon}$ equals the lift of $B\left(v_{0}\right)$ in $T(B(s))$ that contains $\epsilon$. In particular, the tree $T(B(s))_{\text {Aut }(T(B(s))), \epsilon}$ is regular of degree $2(s+1) / 2=s+1$.

Our claim on the values of the scale function follows from Lemma 7. Since the remaining statements made are obvious, the proof of the second claim is complete.

Proof of 3: We first establish the upper bound on the translation length claimed. Recall that the upper bound for the number of vertices of a graph with fundamental group free of rank $n$ and all vertices of degree at least 3 obtained in the proof of Lemma 9 was $2(n-1)$. The longest path without backtracking inside a maximal subtree of a graph with fundamental group free of rank $n$ and all vertices of degree at least 3 therefore is $2(n-1)-1$ and the longest translation length an element of a Schottky basis in the fundamental group over the trivial graph of groups over such a graph can have is $2(n-1)-1+1=2(n-1)$. This establishes the upper bound for the translation length claimed. 
To prove the remainder of the claim, it suffices to prove the existence of the graph $B^{2(n-1)}$ with the properties stated for every integer $n$ at least 2 , since the other statements follow from this. The graph $B^{2(n-1)}$ is constructed as follows. Take the $2(n-1)$ numbers $0, \ldots, 2 n-3$ as the set of vertices of $B^{2(n-1)}$. The number of these vertices is even. Choose, once and for all, a cyclic order on the set of vertices of $B^{2(n-1)}$. Traverse the vertices of $B^{2(n-1)}$ in that cyclic order and connect encountered successive vertices in an alternating fashion by 1 respectively 2 edges. By construction, every vertex of the graph $B^{2(n-1)}$ has degree 3 .

Since the various Schottky bases of the fundamental group of the trivial graph of groups over $B^{2(n-1)}$ are determined by the choice of a maximal subtree in $B^{2(n-1)}$, we determine next all choices of a maximal subtree in $B^{2(n-1)}$. If $T$ is a maximal subtree of $B^{2(n-1)}$, then not every vertex in $B^{2(n-1)}$ can be connected to the previous vertex within $T$ with respect to the chosen cyclic order on the set of vertices, because then $T$ would contain a cycle. Let $v_{0}$ be a vertex of $B^{2(n-1)}$ which is not connected to its predecessor, $v_{2 n-3}$, in the chosen cyclic order. Then the set of edges of $T$ forms a path in cyclic order from $v_{0}$ to $v_{2 n-3}$.

We therefore see that, up to an automorphism of $B^{2(n-1)}$, the maximal subtrees in $B^{2(n-1)}$ are of at most two kinds. If $n$ is at least 3 there are two kinds, depending on whether $v_{2 n-3}$ is connected to $v_{0}$ by 1 or by 2 edges inside $B^{2(n-1)}$, while there is just one kind if $n$ is equal to 2 . Each of the edges connecting $v_{2 n-3}$ to $v_{0}$ that do not belong to $T$ defines an element of the Schottky basis determined by $T$ of translation length $2(n-1)$. We have just seen that there is at least 1 element with such a translation length in every Schottky basis attached to the graph $B^{2(n-1)}$. Since the covering tree $T\left(B^{2(n-1)}\right.$ of $B^{2(n-1)}$ is 3-regular, the scale of such an element with respect to $\operatorname{Aut}\left(T\left(B^{2(n-1)}\right)\right)$ is $2^{2(n-1)}$. We have shown all parts of claim 3 .

The following corollary follows from Proposition 13.

Corollary 14. Let $n$ be an integer which is at least 2 and let $F_{n}$ be the free group of rank $n$. Then, for every prime number, $p$, such that $2 \leq p \leq 2 n-1$, there is a discrete, cocompact embedding, $\varphi: F_{n} \rightarrow G$, into a totally disconnected, locally compact group, $G$, and an element, $h_{\varphi}$, of $F_{n}$ such that $p \mid s_{G}\left(\varphi\left(h_{\varphi}\right)\right)$. Furthermore, every prime, $p$, dividing the value of the scale function on an element of $F_{n}$ with respect to any envelope of $F_{n}$ satisfies $2 \leq p \leq 2 n-1$.

From Proposition 13 we also obtain estimates for the scale volume of the free group of rank at least 2 .

Corollary 15. Let $n$ be an integer which is at least 2 , and let $F_{n}$ be the free group of rank $n$. Then the scale volume, $s-\operatorname{vol}\left(F_{n}\right)$, of $F_{n}$ satisfies the inequalities

$$
(2 n-1)^{n} \leq s-\operatorname{vol}\left(F_{n}\right) \leq(2 n-1)^{2 n(n-1)}
$$

Proof. The stated lower bound follows from part 1 of Proposition 13. The upper bound follows from parts 1 and 3 of Proposition 13.

The gap between the upper and lower bounds in the estimate for $s$-vol $\left(F_{n}\right)$ in Corollary 15 grows fast, and both bounds are very far from the truth. Some experimentation led me to believe that, asymptotically, as $n$ goes to infinity, the value of $s-\operatorname{vol}\left(F_{n}\right)$ is $2^{n\left(2 \log _{2}(n / 3)+3\right)}$. However, I have been forced to revise my initial impression that it is straightforward to determine the asymptotics of the sequence $\left(s-\operatorname{vol}\left(F_{n}\right)\right)_{n \geq 2}$. 


\section{References}

Ahl02. A. Reiter Ahlin. The large scale geometry of products of trees. Geom. Dedicata, 92:179-184, 2002. Dedicated to John Stallings on the occasion of his 65th birthday.

Bas93. Hyman Bass. Covering theory for graphs of groups. Journal of pure and applied Algebra, 89(12):3-47, 1993.

BM97. Marc Burger and Shahar Mozes. Finitely presented simple groups and products of trees. $C . R$. Acad. Sci. Paris Sér. I Math., 324(7):747-752, 1997.

BW06. Udo Baumgartner and George A. Willis. The direction of an automorphism of a totally disconnected locally compact group. Math. Z., 252:393-428, 2006.

Fur01. A. Furman. Mostow-Margulis rigidity with locally compact targets. Geom. Funct. Anal., 11(1):3059, 2001.

Lub91. Alexander Lubotzky. Lattices in rank one Lie groups over local fields. Geom. Funct. Anal., 1:405-431, 1991.

Möl. Rögnvaldur G. Möller. Analogues of Cayley graphs for topological groups. preprint, February 2006.

MSW03. Lee Mosher, Michah Sageev, and Kevin Whyte. Quasi-actions on trees. I. Bounded valence. Ann. of Math. (2), 158(1):115-164, 2003.

Ser80. Jean-Pierre Serre. Trees. Springer Verlag, 1980. original french edition "Arbres, amalgames, $S L_{2}$ " was published as Astérisque 46 (by Société Mathématique de France) in 1977.

Wil01a. George A. Willis. Further properties of the scale function on a totally disconnected locally compact group. J. Algebra, 237:142-164, 2001.

Wil01b. George A. Willis. The number of prime factors of the scale function on a compactly generated group is finite. Bull. London Math. Soc., 33(2):168-174, 2001.

Wil04. George A. Willis. A canonical form for automorphisms of totally disconnected locally compact groups. In Random walks and geometry, pages 295-316. Walter de Gruyter GmbH \& Co. KG, Berlin, 2004. 